\title{
生源启发下的喜树碱的简洁形式合成
}

\author{
李远志 朱孟倩 徐 亮* \\ (四川大学华西药学院 天然药物系暨教育部靶向药物重点实验室 成都 610041)
}

\begin{abstract}
摘要 以色胺和方便易得的乙基戊烯二酫盐为起始物，以简洁的步骤(最终实现喜树碱全合成共 9 步反应)完成了喜树 碱的生源启发下的无保护基形式合成. 合成路线涉及关键的 Pictet-Spengler 反应, 分子内氧杂 Diels-Alder 反应高效构建 单萜吲哚中间体, 以及 Winterfeldt 高效仿生氧化吲哚合成喹啉酮结构.
\end{abstract}

关键词 喜树碱; Pictet-Spengler 反应; 氧杂 Diels-Alder 反应; Winterfeldt 氧化; 形式合成

\section{A Concise Biogenetically Inspired Formal Synthesis of Camptothecin}

\author{
Li, Yuanzhi Zhu, Mengqian $\quad \mathrm{Xu}$, Liang* \\ (Key Laboratory of Drug Targeting, Ministry of Education, and Department of Chemistry of Medicinal Natural Products, West \\ China College of Pharmacy, Sichuan University, Chengdu 610041)
}

\begin{abstract}
A concise biogenetically inspired formal synthesis of camptothecin without use of protecting groups has been developed starting from tryptamine and easily prepared ethyl glutaconaldehyde salt. The synthesis features the key Pictet-Spengler reaction, efficient intramolecular oxa-Diels-Alder reaction to construct heptacyclic monoterpenoid indole alkaloid intermediate, as well as the following Winterfeldt biomimetic oxidation to form quinolinone moiety from indole skeleton.

Keywords Camptothecin; Pictet-Spengler reaction; oxa-Diels-Alder reaction; Winterfeldt oxidation; formal synthesis
\end{abstract}

喜树碱(camptothecin, 1)是 Wani 和 Wall 等 ${ }^{[1]}$ 在 20 世纪 60 年代从源自中国的特有植物喜树(Camtoptheca acuminata)中率先分离鉴定的一种结构新颖的喹啉生物 碱化合物(图 1). 其独特的五环内酯结构和显著的动物 体外和体内抗肿瘤活性在其发现之初就引起了制药界 的高度关注. 然而进一步的药学研究发现天然喜树碱具 有水溶性差、内酯环不稳定导致失活以及副作用大等缺 点 ${ }^{[2]}$, 从而限制了喜树碱的直接临床应用. 喜树碱的作 用机制是通过与肿瘤细胞中高度活跃的拓扑异构酶 I 特 异性结合并抑制其活性, 从而引起 DNA 双链断裂而发 挥细胞毒作用 ${ }^{[3]}$. 这一独特作用机制的阐明使喜树碱在 1980 年代再次引发学术界和制药界的研究热潮 ${ }^{[4]}$. 以喜 树碱作为先导化合物开发高效低毒的喜树碱衍生物成 为新的研究热点. 经过几十年的发展, 已合成了众多喜 树碱结构修饰的类似物, 并评价了其构效关系. 迄今为 止已有伊立替康(irinotecan, 2) ${ }^{[5]}$ 、拓扑替康(topotecan, 3) ${ }^{[6]}$ 和贝洛替康 (belotecan, 4) ${ }^{[7]}$ 三个喜树碱类药物被成 功开发上市, 在临床上被广泛用于结直肠癌、卵巢

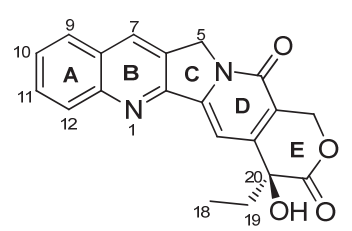<smiles>CC[C@]1(O)C(=O)OCc2c1cc1n(c2=O)Cc2cc3c(CN(C)C)c(O)ccc3nc2-1</smiles>

topotecan (3)

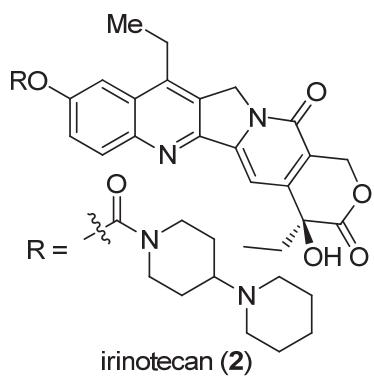<smiles>CC(C)NCCc1c2c(nc3ccccc13)-c1cc3c(c(=O)n1CN2C(C)(C)C)COC(=O)C3(O)O</smiles>

belotecan (4)

图 1 喜树碱及其衍生物

Figure 1 Camptothecin and its derivatives

癌和小细胞肺癌等多种恶性肿瘤的治疗.

* Corresponding author. E-mail: liangxu@scu.edu.cn

Received February 20, 2021; revised March 4, 2021; published online March 25, 2021.

Project supported by the National Natural Science Foundation of China (No. 21871190).

国家自然科学基金(No. 21871190)资助项目. 
尽管通过结构修饰的方式开发喜树碱类衍生物取 得了巨大的成功, 然而天然资源的短缺和环境问题使得 从植物中直接分离获取喜树碱用于制备活性药物并非 一个可以持续的方式, 因此, 发展经济高效的从头合成 喜树碱及其衍生物的全合成路线具有重要的意义. 自 1971 年 Stork 小组 ${ }^{[8]}$ 完成喜树碱的首次全合成以来, 有 关喜树碱的全合成研究取得了重要的进展 ${ }^{[9]}$. 生源启发 下的天然产物仿生合成一直是发展高效天然产物全合 成路线的重要途经之一 ${ }^{[10]}$. 基于喜树碱可能来自于单 萜吲哚生物碱氧化重排的生物合成机制 ${ }^{[1]}, 1972$ 年, Winterfeldt 等 ${ }^{[2]}$ 率先报道了喜树碱的生源启发下的全合 成. 以色胺 5 为起始物, 经 Pictet-Spengler 反应和 Dieckmann 缩合等 5 步反应构建四环吲哚生物碱中间体 6, 再经仿生氧化重排和官能团转化等 7 步反应实现了 喜树碱的全合成. 2000 年, Brown 等 ${ }^{[13]}$ 在多年探索的基 础上, 进一步以色胺 $\mathbf{5}$ 和天然的单萜前体裂环马钱子苷 (secologanin, 7) 为起始原料, 经仿生的高效 PictetSpengler 反应生成单萜吲哚生物碱异胡豆苷(strictosidine), 进而关环生成五环内酰胺中间体 8, 再经保护、 脱保护及多步氧化还原操作, 以总共 12 步 $3 \%$ 的总收率 实现了喜树碱的全合成(Scheme 1, a).

本研究拟以色胺 $\mathbf{5}$ 和更为方便易得的共轭烯醛盐 $\mathbf{9}$
为起始物, 设计经关键的 Pictet-Spengler 反应和分子内 氧杂 Diels-Alder 反应构建吲哚中间体 $\mathbf{1 0}$, 再经 Winterfeldt 吲哚仿生氧化反应合成已知五环喹啉中间体 11, 期望发展一条更为简洁的仿生无保护基合成喜树碱 的路线(Scheme 1,b).

\section{1 结果与讨论}

合成研究首先从制备乙基戊烯二醛盐中间体 9 开始 (Scheme 2). 参考 Marazano 小组 ${ }^{[14]}$ 和 Poupon 小组 ${ }^{[15]}$ 报 道的类似戊烯二醛盐的成熟合成方法，以便宜和商业易 得的 3-乙基吡啶 12 为起始物, 首先与 2,4-二硝基氯苯 13 在回流条件下定量生成相应的吡啶盐 14 (也被称为 Zincke 盐), 随后 14 与二甲胺在乙醇水溶液中回流生成 胺基戊二烯醛(也被称为 Zincke 醛)中间体, 所得粗产品 无需纯化直接在 $\mathrm{KOH}$ 条件下发生碱水解, 共经两步以 $75 \%$ 的总产率方便快捷地以 $10 \mathrm{~g}$ 以上的规模实现了醛 9 制备.

在完成制备醛 9 的基础上，接下进行关键的单萜吲 哚五环中间体 17 的构建. 如 Scheme 3 所示, 色胺 5 首 先和 9 在三氟乙酸的作用下顺利发生 Pictet-Spengler 反 应 ${ }^{[16]}$, 所得四氢 $\beta$-卡波林中间体不经分离, 经简单萃取 后直接与 3-乙氧基丙烯酰氯 15 发生酰化反应，制得氧

(a) Previously reported biogenetically patterned total synthesis of camptothecin

Winterfeldt 1972

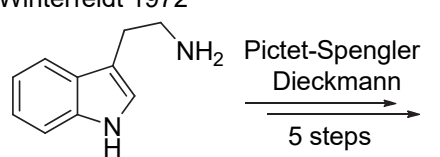

5

Brown 2000
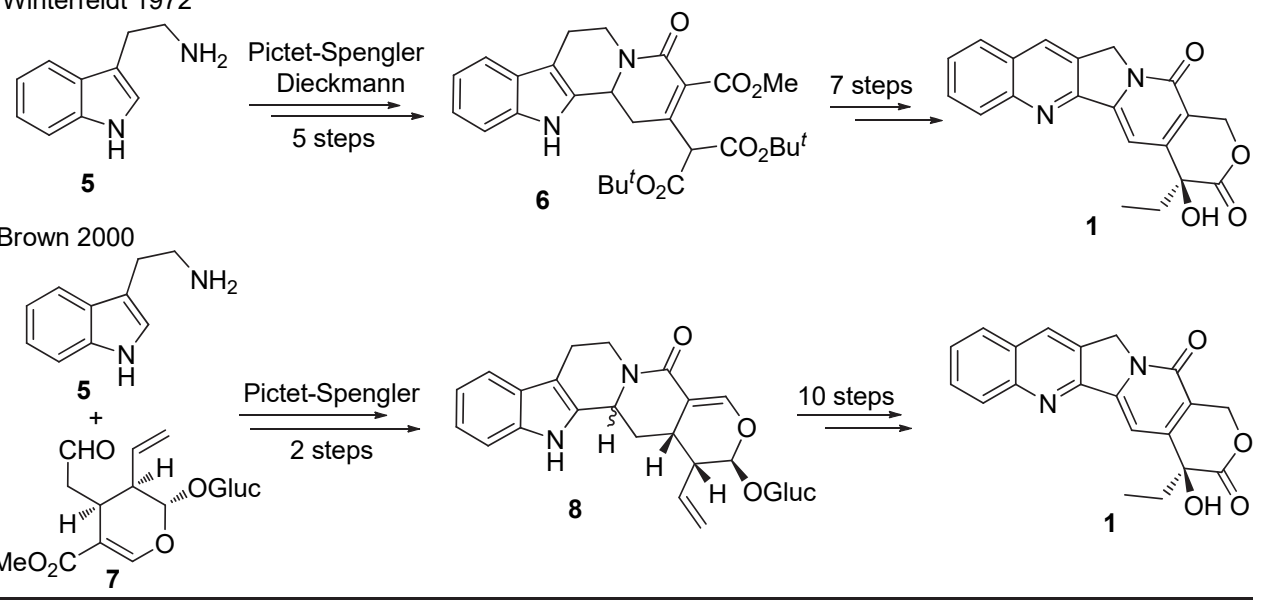

(b) This work
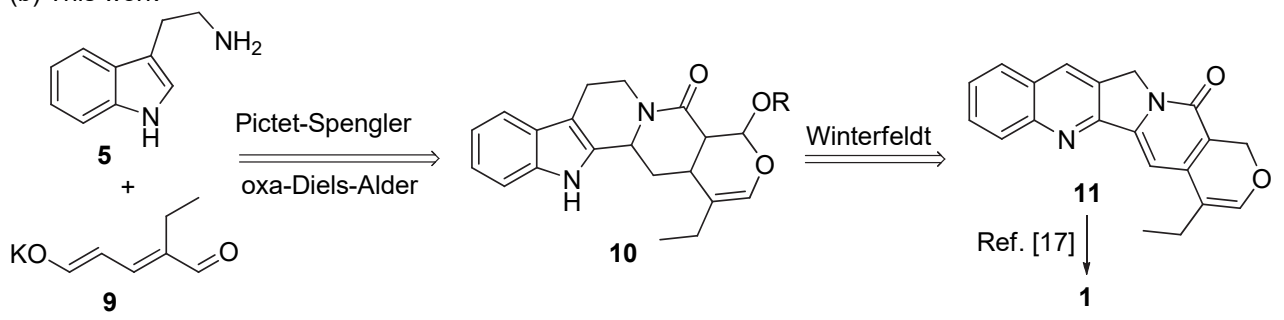

图式 1 已报道的喜树碱的生源启发全合成路线和我们的合成设计

Scheme1 (a) Previously reported biogenetically patterned total synthesis of camptothecin and (b) our synthetic plan 


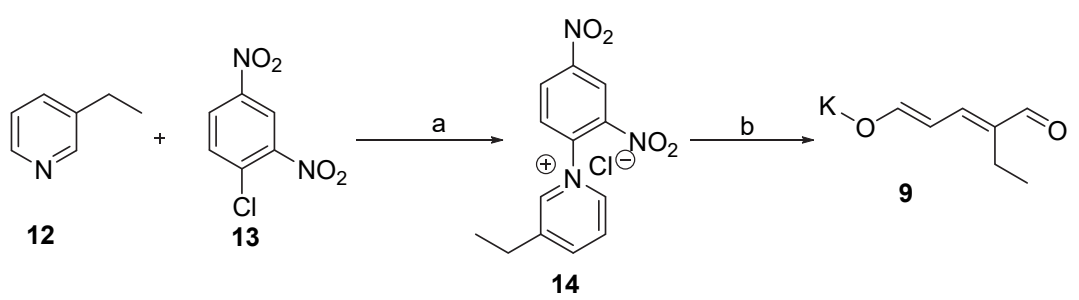

Reagents and conditions: (a) acetone, reflux; (b) dimethylamine, $\mathrm{EtOH} / \mathrm{H}_{2} \mathrm{O}$, reflux; then $\mathrm{KOH}, \mathrm{THF} / \mathrm{MeOH}$, reflux, $75 \%$ for two steps.

图式 2 2-乙基戊烯二醛盐(9)的制备

Scheme 2 Preparation of 2-ethylglutaraldehyde salt (9)

杂 Diles-Alder (D-A)反应前体 16; 接下来以均三甲苯作 为溶剂, 进行热力学条件下的分子内氧杂 Diles-Alder 反 应的尝试 ${ }^{[17]}$, 然而在该条件下反应结果较为复杂, 仅能 以 36\%的收率制得 DE 环反式绸合的关环产物 17 和其 它一些结构未能鉴定的副产物(令人惊讶的是, 这些副 产物经初步鉴定并非化合物 $\mathbf{1 7}$ 的立体异构体). 化合物 17 的立体化学是根据路线后期的喹啉衍生物 20 与文献 [17]对比而确定. 尝试其它高沸点溶剂如氯苯、甲苯和 乙二醇二甲醚等也未能进一步提高反应收率. 我们进而 参考 $\mathrm{Tu}$ 小组 ${ }^{[18]}$ 最近报道的类似氧杂 D-A 方法进行路易 斯酸促进该反应的条件篮选, 结果发现化合物 16 在 1,2二氯乙烷中与 1.5 equiv. 溴化锌回流反应, 能以较高产率 (72\%)得到单一的反式异构体 17 . 在高效完成五环单萜 吲哚中间体 17 的制备后, 接下来进行关键的吲哚仿生 氧化构建喹啉结构, 进而实现喜树碱最终形式合成的研 究. 首先, 17 在经典的 Winterfeldt 仿生氧化条件下 $\left[\mathrm{KO} t-\mathrm{Bu}, \mathrm{O}_{2}, N, N \text {-二甲基甲酰胺(DMF) }\right]^{[12]}$, 能以 $50 \%$ 的 收率转化成喹啉酮 18. 进一步优化碱的种类发现, 以氢 化钠作碱 ${ }^{[19]}$ 能将该关键反应的收率提高至 $75 \%$. 试图通 过二氯亚砜氯代 ${ }^{[12-13]}$ 的方式将 $\mathbf{1 8}$ 中的喹啉酮结构转化 为喹啉单元, 结果发现由于分子中对酸敏感的缩醛结构 的存在使得反应变得复杂, 因此采用更温和的碱性条 件. 将化合物 18 在三乙胺和 4-二甲氨基吡啶(DMAP)条 件下与 $N$ - 苯基双 (三氟甲磺酰亚胺) 反应顺利制得喹啉三 氟磺酰酯化合物 19. 随后 19 再经醋酸钯催化的甲酸还 原, 顺利脱除三氟甲磺酰基, 以 $90 \%$ 的收率实现喹啉中 间体 20 的制备 ${ }^{[20]} .20$ 经 2,3-二氯-5,6-二氧-1,4-苯醌 (DDQ)氧化芳香化, 和三乙基氢硅烷还原脱除乙氧基, 以两步 75\%的收率制得喜树碱前体化合物 11.20 和 11 与 Yao 小组和 Gao 小组 ${ }^{[17,21]}$ 报道的化合物的核磁数据完 全一致. 11 经过 Sharpless 不对称双羟基化和 $\mathrm{I}_{2}-\mathrm{CaCO}_{3}$ 氧化两步文献报道的已知反应 ${ }^{[8]}$, 即可容易地实现喜树 碱 1 的全合成.

\section{2 结论}

以色胺和方便易得的乙基戊烯二醛盐为起始物, 以
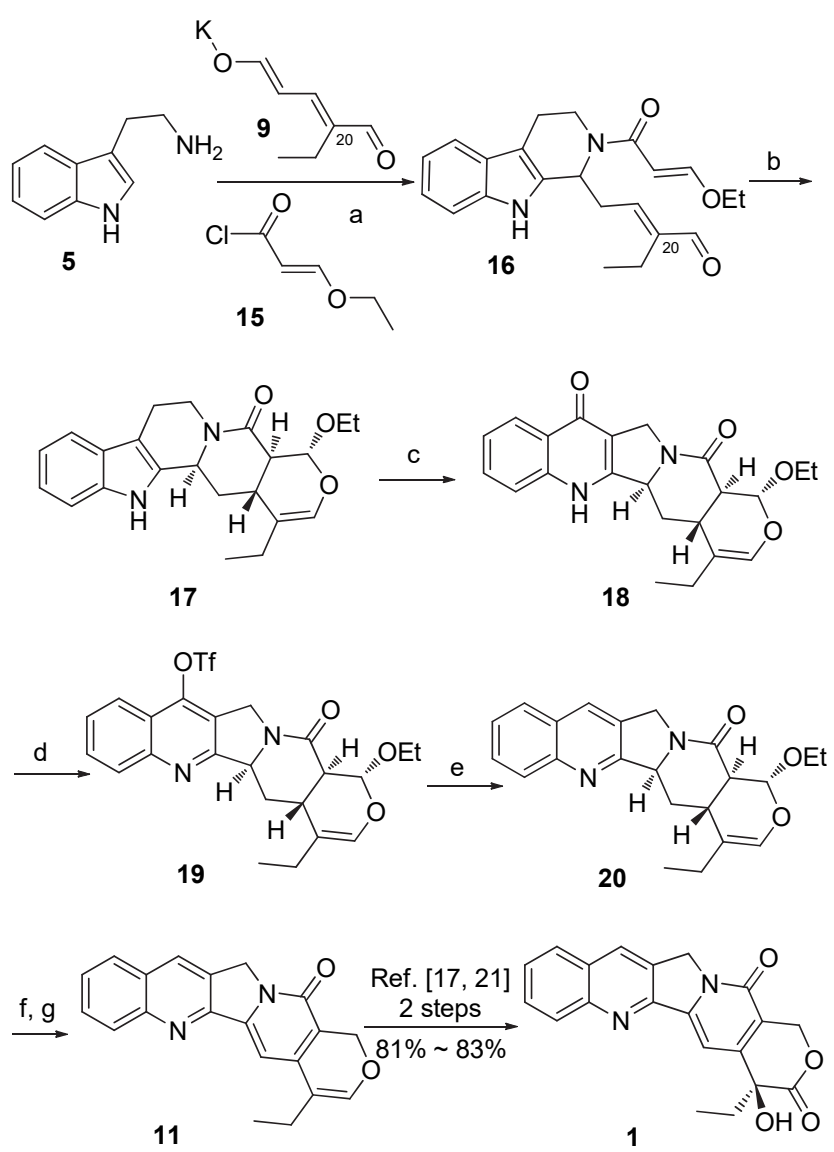

Reagents and conditions: (a) 9, TFA, DCM, r.t. to $-5{ }^{\circ} \mathrm{C}$, then $\mathbf{1 5}$, $\mathrm{NEt}_{3}, \mathrm{DCM}$, r.t., $80 \%$; (b) $\mathrm{ZnBr}_{2}, \mathrm{DCE}$, reflux, $72 \%$; (c) $\mathrm{O}_{2}, \mathrm{NaH}$, DMF, 75\%; (d) $\mathrm{Tf}_{2} \mathrm{NPh}$, DMAP, $\mathrm{NEt}_{3}$, DCM, r.t., 86\%; (e) $\mathrm{Pd}(\mathrm{OAc})_{2}$, $\mathrm{DPPF}, \mathrm{HCOOH}, \mathrm{NEt}_{3}$, dioxane, $80{ }^{\circ} \mathrm{C}, 90 \%$; (f) $\mathrm{DDQ}$, dioxane, r.t.; (g) $\mathrm{Et}_{3} \mathrm{SiH}, \mathrm{BF}_{3} \cdot \mathrm{Et}_{2} \mathrm{O}, \mathrm{DCM}$, r.t., $75 \%$ over two steps.

图式 3 合成关键中间体 11 和喜树碱(1)形式全合成 Scheme 3 Synthesis of key intermediate $\mathbf{1 1}$ and formal total synthesis of camptothecin (1)

简洁的步骤(最终实现喜树碱全合成共 9 步反应)完成了 喜树碱的生源启发下的无保护基的形式合成. 合成路线 涉及关键的 Pictet-Spengler 反应, 分子内氧杂 DielsAlder 反应高效构建单萜吲哚中间体, 以及 Winterfeldt 高效仿生氧化吲哚合成喹啉酮. 该路线不仅提供了一种 更为简洁的生源模式合成喜树碱的方法，也为进一步制 
备 $\mathrm{AB}$ 环或 $\mathrm{E}$ 环的喜树碱衍生物, 以及研究其相应构效 关系奠定了良好的基础，相关工作仍在进行之中.

\section{3 实验部分}

\section{1 仪器与试剂}

旋光用 Perkin Polarimeter 341 型旋光光度测定仪测 定; 核磁共振用 Varian Unity NOVA-400/54 核磁共振仪 测定 (TMS 为内标). HRMS 使用 Bruker Daltonics Bio-TOF-Q 质谱仪测定. 未有特别说明反应均在干燥氞 气保护下进行. 所有实验药品均为市售分析纯试剂, 使 用前未进行纯化处理. 1,4-二氧六环使用前经过钠/二苯 甲酮除水处理; $N, N$-二甲基甲酰胺、1,2-二氯乙烷和二氯 甲烷使用前经过氢化钻除水处理; 薄层色谱(TLC) GF254 硅胶板购自烟台江友硅胶开发有限公司，用 $\mathrm{I}_{2}$ 、 高锰酸钾溶液或苂光显色; 柱层析用硅胶(200 300 目) 购自青岛海洋化工厂。

\section{2 实验方法}

\subsubsection{2-乙基戊烯二醛盐(9)的合成}

室温下, 将 3-乙基吡啶 $(\mathbf{1 2}, 11.2 \mathrm{~mL}, 98 \mathrm{mmol})$ 与 2,4-二硝基氯苯(13, $20 \mathrm{~g}, 98 \mathrm{mmol}$ )溶于丙酮(120 mL)中, 之后移入事先预热好的 $60{ }^{\circ} \mathrm{C}$ 油浴中回流 $10 \mathrm{~h}$, 反应毕, 将混悬的反应液移至室温冷却, 过滤沉淀, 并用丙酮 $(60 \mathrm{~mL})$ 洗涤沉淀 2 次, 收集沉淀, 得吡啶盐中间体 $\mathbf{1 4}$ $(30 \mathrm{~g})$. 将 14 溶于水 $(240 \mathrm{~mL})$ 与乙醇 $(240 \mathrm{~mL})$ 的混合溶 剂中, 移入事先预热好的 $90{ }^{\circ} \mathrm{C}$ 油浴, 并在回流状态下 滴加入二甲胺乙醇溶液 $(5.6 \mathrm{~mol} / \mathrm{L}, 34 \mathrm{~mL}, 192 \mathrm{mmol})$, 搅拌反应 $8 \mathrm{~h}$, 至 TLC 检测显示反应结束. 通过减压浓 缩反应液, 再向浓缩体系中加入水 $(120 \mathrm{~mL})$, 搅拌 $1 \mathrm{~h}$, 二氯甲烷 $(240 \mathrm{~mL} \times 2)$ 萃取, 分离有机层, 饱和食盐水 $(80 \mathrm{~mL})$ 洗涤, 无水硫酸钠干燥, 减压蒸馏浓缩, 得胺基 戊二烯醛中间体 $(13 \mathrm{~g})$. 中间体未进一步纯化, 直接将 其溶解于四氢呋喃 $(240 \mathrm{~mL})$ 中, 室温下将氢氧化钾 (4.4 $\mathrm{g}, 78 \mathrm{mmol})$ 的甲醇 $(20 \mathrm{~mL})$ 溶液滴加入反应液, 之后移 入事先预热好的 $68{ }^{\circ} \mathrm{C}$ 油浴中回流反应 $10 \mathrm{~h}$. 反应毕, 将反应液移至室温, 加入乙梄 $(240 \mathrm{~mL})$ 搅拌 $30 \mathrm{~min}$, 过 滤, 收集沉淀. 然后将沉淀溶于无水乙醇 $(400 \mathrm{~mL})$ 中, 于 $-10{ }^{\circ} \mathrm{C}$ 下摚拌 $30 \mathrm{~min}$, 移至室温, 过滤, 收集滤液, 通过减压蒸馏浓缩得淡黄色固体 9 (11 g, 75\%). m.p. $140 \sim 142{ }^{\circ} \mathrm{C} ;{ }^{1} \mathrm{H}$ NMR $\left(400 \mathrm{MHz}, \mathrm{D}_{2} \mathrm{O}\right) \delta: 8.53(\mathrm{~d}, J=$ $10.0 \mathrm{~Hz}, 1 \mathrm{H}), 8.45(\mathrm{~s}, 1 \mathrm{H}), 7.38(\mathrm{~d}, J=13.2 \mathrm{~Hz}, 1 \mathrm{H}), 5.78$ (dd, $J=13.2 \mathrm{~Hz}, 10.0,1 \mathrm{H}), 2.21$ (q, $J=7.6 \mathrm{~Hz}, 2 \mathrm{H}), 0.93$ $(\mathrm{t}, J=7.6 \mathrm{~Hz}, 3 \mathrm{H}) ;{ }^{13} \mathrm{C}$ NMR $\left(101 \mathrm{MHz}, \mathrm{D}_{2} \mathrm{O}\right) \delta: 187.8$, 186.3, 164.7, 125.2, 107.1, 16.5, 12.4; ESI-HRMS calcd for $\mathrm{C}_{7} \mathrm{H}_{9} \mathrm{O}_{2}[\mathrm{M}-\mathrm{K}]^{-}$125.0608, found 125.0628 .
3.2.2 (+/ - )-(E)-4-(2-((E)-3-乙氧基丙烯酰基)-2,3,4, 9-四氢- $1 H$-吡啶并 $[3,4-b]$ 吲哚-1-基)-2-乙基-2-烯醛 (16)的合成

将色胺 5 (1 g, $6 \mathrm{mmol})$ 溶解于二氯甲烷 $(100 \mathrm{~mL})$ 中, 室温下滴加入三氟乙酸 $(0.45 \mathrm{~mL}, 6 \mathrm{mmol})$, 待反应液由 淡黄色变为深绿色时, 再将化合物 $9(1 \mathrm{~g}, 6 \mathrm{mmol})$ 一次 性加入反应液, 室温下搅拌反应至薄层色谱检测显示反 应结束. 再将反应液移至 $-5{ }^{\circ} \mathrm{C}$ 的低温中, 滴加入三氟 乙酸 $(1.8 \mathrm{~mL}, 24 \mathrm{mmol})$, 继续摚拌反应 $1.5 \mathrm{~h}$ 至薄层色谱 检测显示反应结束. 再将反应液移至室温, 加入饱和碳 酸氢钠水溶液至无气泡产生, 分离有机层, 饱和食盐水 $(20 \mathrm{~mL})$ 洗涤，无水硫酸钠干燥，有机层通过减压蒸馏浓 缩，得四氢 $\beta$-卡波林中间体 $(1.5 \mathrm{~g})$, 中间体未经进一步 纯化. 室温下，向粗品中间体的二氯甲烷 $(60 \mathrm{~mL})$ 溶液中 依次加入三乙胺 $(1 \mathrm{~mL}, 7.4 \mathrm{mmol}$ )和 3-乙氧基丙烯酰氯 $(15,0.6 \mathrm{~mL}, 5 \mathrm{mmol})$, 继续摚拌反应至 TLC 检测显示反 应结束, 加水 $(20 \mathrm{~mL})$ 淬灭反应，二氯甲烷 $(20 \mathrm{~mL} \times 2)$ 萃 取, 分离有机层, 无水硫酸钠干燥, 有机层通过减压蒸 馏浓缩, 所得粗品经闪式硅胶柱层析纯化(洗脱剂: 石 油醚/乙酸乙酯, $V: V=2: 1)$ 得淡黄色固体 $\mathbf{1 6}(1.7 \mathrm{~g}$, $80 \%)$. m.p. $161 \sim 163{ }^{\circ} \mathrm{C} ;{ }^{1} \mathrm{H}$ NMR $\left(400 \mathrm{MHz}, \mathrm{CDCl}_{3}\right) \delta$ : $9.26(\mathrm{~s}, 1 \mathrm{H}), 8.60(\mathrm{~s}, 1 \mathrm{H}), 7.65$ (d, $J=11.6 \mathrm{~Hz}, 1 \mathrm{H}), 7.47$ (d, $J=8.0 \mathrm{~Hz}, 1 \mathrm{H}), 7.33(\mathrm{~d}, J=8.0 \mathrm{~Hz}, 1 \mathrm{H}), 7.18(\mathrm{t}, J=8.0$ $\mathrm{Hz}, 1 \mathrm{H}), 7.11(\mathrm{t}, J=8.0 \mathrm{~Hz}, 1 \mathrm{H}), 6.54(\mathrm{t}, J=7.2 \mathrm{~Hz}, 1 \mathrm{H})$, $6.09(\mathrm{~s}, 1 \mathrm{H}), 5.78(\mathrm{~d}, J=11.6 \mathrm{~Hz}, 1 \mathrm{H}), 4.17 \sim 4.07(\mathrm{~m}$, $1 \mathrm{H}), 3.99$ (q, $J=7.2 \mathrm{~Hz}, 2 \mathrm{H}), 3.51 \sim 3.44(\mathrm{~m}, 1 \mathrm{H}), 3,04 \sim$ $2.90(\mathrm{~m}, 2 \mathrm{H}), 2.88 \sim 2.79(\mathrm{~m}, 2 \mathrm{H}), 2.18(\mathrm{q}, J=7.2 \mathrm{~Hz}$, $2 \mathrm{H}), 1.36(\mathrm{t}, J=7.2 \mathrm{~Hz}, 3 \mathrm{H}), 0.86(\mathrm{t}, J=7.2 \mathrm{~Hz}, 3 \mathrm{H}) ;{ }^{13} \mathrm{C}$ NMR $\left(101 \mathrm{MHz}, \mathrm{CDCl}_{3}\right) \delta: 194.6,167.0,162.5,148.7$, $146.5,136.1,132.9,126.3,121.8,119.4,117.8,111.0$, 108.0, 95.2, 67.6, 48.4, 40.5, 33.7, 22.0, 17.0, 14.5, 13.0; ESI-HRMS calcd for $\mathrm{C}_{22} \mathrm{H}_{27} \mathrm{~N}_{2} \mathrm{O}_{3}[\mathrm{M}+\mathrm{H}]^{+} 367.2016$, found 367.2021 .

3.2.3 (4R, $4 \mathrm{a} R, 13 \mathrm{~b} S, 14 \mathrm{a} R)$-4-乙氧基-1-乙基-4,4a,7, $8,13,13 \mathrm{~b}, 14,14 \mathrm{a}$-八氢 $-5 H$ - 吲哚并 $[2,3-\mathrm{a}]$ 吡喃并 $[3,4-g]$ 喹啉-5-酮(17)的合成

室温下，向化合物 $\mathbf{1 6}$ (200 mg, $0.55 \mathrm{mmol})$ 的 1,2-二 氯乙烷 $(54 \mathrm{~mL})$ 溶液中, 加入溴化锌 $(184 \mathrm{mg}, 0.83$ $\mathrm{mmol})$, 之后移入事先预热好的 $85{ }^{\circ} \mathrm{C}$ 油浴中回流反应 至 TLC 检测显示反应结束, 加入水 $(15 \mathrm{~mL})$ 淬灭反应, 二氯甲烷 $(20 \mathrm{~mL} \times 2)$ 萃取, 饱和食盐水 $(10 \mathrm{~mL})$ 洗涤, 分 离有机层, 无水硫酸钠干燥, 有机层通过减压蒸馏浓缩, 所得粗品经闪式硅胶柱层析纯化(洗脱剂: 石油醚/乙酸 乙酯, $V: V=2 ： 1$ )得淡黄色固体 17 (145 mg, 72\%). m.p. $181 \sim 183{ }^{\circ} \mathrm{C} ;{ }^{1} \mathrm{H}$ NMR $\left(600 \mathrm{MHz}, \mathrm{CD}_{3} \mathrm{OD}\right) \delta: 7.38(\mathrm{~d}$, 
$J=8.0 \mathrm{~Hz}, 1 \mathrm{H}), 7.30(\mathrm{~d}, J=8.0 \mathrm{~Hz}, 1 \mathrm{H}), 7.07$ (t, $J=8.0$ $\mathrm{Hz}, 1 \mathrm{H}), 6.98$ (t, $J=8.0 \mathrm{~Hz}, 1 \mathrm{H}), 6.12(\mathrm{~s}, 1 \mathrm{H}), 5.03$ (d, $J=$ $4.8 \mathrm{~Hz}, 1 \mathrm{H}), 4.94(\mathrm{~d}, J=5.6 \mathrm{~Hz}, 1 \mathrm{H}), 4.70(\mathrm{dd}, J=8.4,3.6$ $\mathrm{Hz}, 1 \mathrm{H}), 3.95 \sim 3.84(\mathrm{~m}, 1 \mathrm{H}), 3.82 \sim 3.70(\mathrm{~m}, 1 \mathrm{H}), 3.03$ (td, $J=8.4,2.8 \mathrm{~Hz}, 1 \mathrm{H}), 2.93 \sim 2.87(\mathrm{~m}, 1 \mathrm{H}), 2.68 \sim 2.63$ $(\mathrm{m}, 2 \mathrm{H}), 2.43 \sim 2.39(\mathrm{~m}, 1 \mathrm{H}), 2.30 \sim 2.25(\mathrm{~m}, 1 \mathrm{H}), 2.09 \sim$ $1.98(\mathrm{~m}, 3 \mathrm{H}), 1.24$ (t, $J=4.8 \mathrm{~Hz}, 3 \mathrm{H}), 0.94(\mathrm{t}, J=4.8 \mathrm{~Hz}$, $3 \mathrm{H}) ;{ }^{13} \mathrm{C}$ NMR (150 MHz, $\left.\mathrm{CD}_{3} \mathrm{OD}\right) \delta: 171.9,138.1,137.8$, $134.7,128.4,122.6,120.2,118.8,116.9,112.2,100.4$, 99.9, 65.9, 54.6, 48.7, 43.3, 32.3, 31.7, 22.8, 21.5, 15.7, 13.7; ESI-HRMS calcd for $\mathrm{C}_{22} \mathrm{H}_{27} \mathrm{~N}_{2} \mathrm{O}_{3} \quad[\mathrm{M}+\mathrm{H}]^{+}$ 367.2016, found 367.2021 .

3.2.4 ( $1 R, 4 \mathrm{a} R, 5 \mathrm{a} S, 14 \mathrm{a} R)-1$ - 乙氧基-4-乙基- $4 \mathrm{a}, 5,5 \mathrm{a}, 6$, $12,14 \mathrm{a}$ - 六氢 $-1 H$-吡喃并 $\left[3^{\prime}, 4^{\prime}: 6,7\right]$ 吲哚并 $[1,2-b]$ 喹啉11,14-二酮(18)的合成

于室温下, 将化合物 $\mathbf{1 7}$ (100 mg, $0.28 \mathrm{mmol}$ )溶解于 DMF ( $8 \mathrm{~mL})$ 中, 加入氢化钠 $(24 \mathrm{mg}, 0.56 \mathrm{mmol})$. 将反应 体系置换成氧气, 并在氧气球下, 搅拌反应 $30 \mathrm{~min}$. 经 TLC 检测显示反应结束, 通过减压蒸馏浓缩反应液, 所 得粗品经闪式硅胶柱层析纯化(洗脱剂: 二氯甲烷/甲醇, $V: V=20: 1$ )得白色固体 18 (80 mg, 75\%). m.p. 200 $202{ }^{\circ} \mathrm{C} ;{ }^{1} \mathrm{H}$ NMR (400 MHz, DMSO) $\delta$ : $12.24(\mathrm{~s}, 1 \mathrm{H})$, 8.14 (d, $J=8.0 \mathrm{~Hz}, 1 \mathrm{H}), 7.68$ (d, $J=8.0 \mathrm{~Hz}, 1 \mathrm{H}), 7.60$ (d, $J=8.0 \mathrm{~Hz}, 1 \mathrm{H}), 7.35$ (s, 1H), 6.18 (s, 1H), 5.30 (t, $J=8.0$ $\mathrm{Hz}, 1 \mathrm{H}), 4.99(\mathrm{~d}, J=8.0 \mathrm{~Hz}, 1 \mathrm{H}), 4.53(\mathrm{~d}, J=16.0 \mathrm{~Hz}$, $1 \mathrm{H}), 4.30(\mathrm{~d}, J=16.0 \mathrm{~Hz}, 1 \mathrm{H}), 3.92 \sim 3.86(\mathrm{~m}, 1 \mathrm{H}), 3.75 \sim$ $3.67(\mathrm{~m}, 1 \mathrm{H}), 2.63 \sim 2.54(\mathrm{~m}, 1 \mathrm{H}), 2.49 \sim 2.43(\mathrm{~m}, 1 \mathrm{H})$, $2.27 \sim 2.21(\mathrm{~m}, 2 \mathrm{H}), 1.96(\mathrm{q}, J=7.2 \mathrm{~Hz}, 2 \mathrm{H}), 1.18(\mathrm{t}, J=$ $7.2 \mathrm{~Hz}, 3 \mathrm{H}), 0.93$ (t, $J=7.2 \mathrm{~Hz}, 3 \mathrm{H}) ;{ }^{13} \mathrm{C}$ NMR $(101 \mathrm{MHz}$, DMSO) $\delta: 172.9,168.5,150.4,140.5,135.6,131.5,125.1$, 124.7, 123.1, 118.4, 115.2, 112.5, 98.4, 64.4, 57.5, 47.2, 44.8, 31.8, 29.7, 22.0, 15.1, 13.0; ESI-HRMS calcd for $\mathrm{C}_{22} \mathrm{H}_{25} \mathrm{~N}_{2} \mathrm{O}_{4}[\mathrm{M}+\mathrm{H}]^{+}$381.1809, found 381.1819.

$3.2 .5(1 R, 4 \mathrm{a} R, 5 \mathrm{a} S, 14 \mathrm{a} R)-1$ - 乙氧基-4-乙基-14-氧代$4 \mathrm{a}, 5,5 \mathrm{a}, 12,14,14 \mathrm{a}$ - 六氢 $-1 H$ - 吡喃并 [3', 4':6,7] 吲哚并 [1,2- $b]$ 喹啉-11-基三氟甲烷磺酸盐(19)的合成

在室温下, 将化合物 18 (145 mg, $0.38 \mathrm{mmol})$ 混悬于 二氯甲烷 $(30 \mathrm{~mL})$ 中, 超声 $5 \mathrm{~min}$ 使反应液呈均匀的混悬 体系, 然后依次加入 $N$-苯基双 (三氟甲烷磺酰)亚胺(205 $\mathrm{mg}, 0.57 \mathrm{mmol})$ 、三乙胺 $(0.15 \mathrm{~mL}, 1 \mathrm{mmol})$ 和 4- $N, N$-二甲 基吡啶 $(28 \mathrm{mg}, 0.23 \mathrm{mmol}$ ). 搅拌反应 $3 \mathrm{~h}$ 后, 经 TLC 检 测显示反应结束, 加水 $(10 \mathrm{~mL})$ 淬灭反应, 二氯甲烷 $(20$ $\mathrm{mL} \times 3)$ 萃取, 饱和食盐水 $(10 \mathrm{~mL})$ 洗涤, 分出有机相, 无 水硫酸钠干燥, 减压蒸馏浓缩, 所得粗品经闪式硅胶柱 层析纯化(洗脱剂: 石油醚/乙酸乙酯, $V: V=5: 1$ )得白
色固体 19 (164 mg, 86\%). m.p. 253 $255{ }^{\circ} \mathrm{C} ;{ }^{1} \mathrm{H}$ NMR $\left(400 \mathrm{MHz}, \mathrm{CDCl}_{3}\right) \delta: 8.19(\mathrm{~d}, J=8.0 \mathrm{~Hz}, 1 \mathrm{H}), 8.11(\mathrm{~d}, J=$ $8.0 \mathrm{~Hz}, 1 \mathrm{H}), 7.86(\mathrm{t}, J=8.0 \mathrm{~Hz}, 1 \mathrm{H}), 7.73(\mathrm{t}, J=8.0 \mathrm{~Hz}$, $1 \mathrm{H}), 6.18(\mathrm{~s}, 1 \mathrm{H}), 5.34(\mathrm{~d}, J=16.8 \mathrm{~Hz}, 1 \mathrm{H}), 5.17 \sim 5.10$ (m, 2H), $4.82(\mathrm{~d}, J=16.8 \mathrm{~Hz}, 1 \mathrm{H}), 4.07 \sim 3.88(\mathrm{~m}, 2 \mathrm{H})$, $2.58 \sim 2.40(\mathrm{~m}, 4 \mathrm{H}), 2.03(\mathrm{q}, J=7.2 \mathrm{~Hz}, 2 \mathrm{H}), 1.35$ (t, $J=$ $7.2 \mathrm{~Hz}, 3 \mathrm{H}), 0.96$ (t, $J=7.2 \mathrm{~Hz}, 3 \mathrm{H}) ;{ }^{13} \mathrm{C}$ NMR $(101 \mathrm{MHz}$, $\left.\mathrm{CDCl}_{3}\right) \delta: 170.0,164.7,151.0,136.4,131.2,129.4,128.6$, $121.4,121.1,120.5,114.9,98.4,65.9,58.8,47.0,46.3$, 32.7, 31.5, 22.6, 15.4, 13.2; ESI-HRMS calcd for $\mathrm{C}_{23} \mathrm{H}_{24-}$ $\mathrm{F}_{3} \mathrm{~N}_{2} \mathrm{O}_{6} \mathrm{~S}[\mathrm{M}+\mathrm{H}]^{+}$513.1302, found 513.1312.

3.2.6 $(1 R, 4 \mathrm{a} R, 5 \mathrm{a} S, 14 \mathrm{a} R)-1$-乙氧基-4-乙基- $1,4 \mathrm{a}, 5,5 \mathrm{a}$, $12,14 \mathrm{a}$-六氢- $14 H$-吡喃并 $\left[3^{\prime} \cdot 4^{\prime}: 6,7\right]$ 吲哚并 $[1,2-b]$ 喹啉14-酮(20)的合成

室温下，向化合物 19 (30 mg, $0.06 \mathrm{mmol}$ )的 1,4-二 氧六环 $(2 \mathrm{~mL})$ 溶液中, 依次加入醋酸钯 $(1 \mathrm{mg}, 0.001$ $\mathrm{mmol}$ )、1,1'-双(二苯基膦)二茂铁 $(1 \mathrm{mg}, 0.001 \mathrm{mmol}) 、 三$ 乙胺 $(20 \mu \mathrm{L}, 0.18 \mathrm{mmol})$ 和甲酸 $(6 \mu \mathrm{L}, 0.12 \mathrm{mmol})$, 再置 于 $80{ }^{\circ} \mathrm{C}$ 下反应 $1 \mathrm{~h}$. 经 TLC 检测显示反应结束, 加水 $(2$ $\mathrm{mL})$ 淬灭反应, 二氯甲烷 $(\mathrm{DCM})(5 \mathrm{~mL} \times 3)$ 萃取, 分出有 机层, 饱和食盐水 $(5 \mathrm{~mL})$ 洗涤, 无水硫酸钠干燥, 减压 蒸馏浓缩, 所得粗品经闪式硅胶柱层析纯化(洗脱剂: 石油醚/乙酸乙酯, $V: V=3: 1)$ 得白色固体 $20(19 \mathrm{mg}$, 90\%). m.p. $172 \sim 174{ }^{\circ} \mathrm{C}$ (lit. ${ }^{[17]} 172 \sim 174{ }^{\circ} \mathrm{C}$ ); ${ }^{1} \mathrm{H}$ NMR $\left(400 \mathrm{MHz}, \mathrm{CDCl}_{3}\right) \delta: 8.11 \sim 8.09(\mathrm{~m}, 2 \mathrm{H}), 7.84(\mathrm{~d}, J=8.0$ $\mathrm{Hz}, 1 \mathrm{H}), 7.73(\mathrm{t}, J=8.0 \mathrm{~Hz}, 1 \mathrm{H}), 7.57$ (t, $J=8.0 \mathrm{~Hz}, 1 \mathrm{H})$, $6.16(\mathrm{~s}, 1 \mathrm{H}), 5.14 \sim 5.07(\mathrm{~m}, 3 \mathrm{H}), 4.73(\mathrm{~d}, J=16.0 \mathrm{~Hz}$, $1 \mathrm{H}), 4.08 \sim 3.87(\mathrm{~m}, 2 \mathrm{H}), 2.60 \sim 2.42(\mathrm{~m}, 4 \mathrm{H}), 2.05 \sim 1.99$ (m, 2H), 1.35 (t, $J=7.2 \mathrm{~Hz}, 3 \mathrm{H}), 0.95(\mathrm{t}, J=7.2 \mathrm{~Hz}, 3 \mathrm{H})$; ${ }^{13} \mathrm{C} \mathrm{NMR}\left(101 \mathrm{MHz}, \mathrm{CDCl}_{3}\right) \delta: 170.0,162.4,148.1,135.9$, 130.2, 129.4, 128.7, 127.7, 127.4, 126.6, 115.0, 98.2, 65.5, 58.1, 47.5, 46.6, 32.6, 31.3, 22.3, 15.1, 12.9; ESI-HRMS calcd for $\mathrm{C}_{22} \mathrm{H}_{25} \mathrm{~N}_{2} \mathrm{O}_{3}[\mathrm{M}+\mathrm{H}]^{+}$365.1860, found 365.1852 .

3.2.7 4-乙基-1,12-二氢- $14 H$-吡喃并 $\left[3^{\prime}, 4^{\prime}: 6,7\right]$ 狮并 [1,2- $b]$ 喹啉-14-酮(11)的合成

室温条件下, 将化合物 $\mathbf{2 0}$ (19 mg, $0.06 \mathrm{mmol}$ )溶解 于 1,4-二氧六环 $(2 \mathrm{~mL})$ 中, 加入 $\mathrm{DDQ}(24 \mathrm{mg}, 0.12$ $\mathrm{mmol}$ )和催化量的冰乙酸, 搅拌反应 $3 \mathrm{~h}$, 薄层层析检测 至原料反应完全, 通过减压蒸馏浓缩, 残留物经快速柱 层析(洗脱剂: 二氯甲烷/甲醇, $V: V=20: 1$ )得到粗品 中间体 $(17 \mathrm{mg})$, 将中间体溶解于二氯甲烷 $(2 \mathrm{~mL})$ 中, 依 次加入三乙基硅烷 $(25 \mu \mathrm{L}, 0.16 \mathrm{mmol})$ 与三氟化嗍乙醚 $(16 \mu \mathrm{L}, 0.13 \mathrm{mmol})$, 室温下搅拌反应 $2 \mathrm{~h}$. 反应毕, 加水 $(2 \mathrm{~mL})$ 淬灭反应, 二氯甲烷 $(5 \mathrm{~mL} \times 3)$ 萃取, 分离有机 
层, 饱和食盐水 $(4 \mathrm{~mL})$ 洗涤, 无水硫酸钠干燥, 减压蒸 馏浓缩, 所得粗品经闪式硅胶柱层析纯化(洗脱剂: 二 氯甲烷/甲醇, $V: V=80: 1)$ 得淡黄色固体 11 (13 mg, 75\%). m.p. $200 \sim 202{ }^{\circ} \mathrm{C}$; ${ }^{1} \mathrm{H}$ NMR (400 MHz, $\left.\mathrm{CDCl}_{3}\right) \delta$ : 8.33 (s, 1H), $8.21(\mathrm{~d}, J=8.4 \mathrm{~Hz}, 1 \mathrm{H}), 7.90$ (d, $J=7.8 \mathrm{~Hz}$, $1 \mathrm{H}), 7.80$ (t, $J=7.2 \mathrm{~Hz}, 1 \mathrm{H}), 7.63$ (t, $J=7.6 \mathrm{~Hz}, 1 \mathrm{H}), 7.20$ $(\mathrm{s}, 1 \mathrm{H}), 6.66(\mathrm{~s}, 1 \mathrm{H}), 5.25(\mathrm{~s}, 2 \mathrm{H}), 5.17(\mathrm{~s}, 2 \mathrm{H}), 2.45$ (q, $J=7.6 \mathrm{~Hz}, 2 \mathrm{H}), 1.21(\mathrm{t}, J=7.6 \mathrm{~Hz}, 3 \mathrm{H}) ;{ }^{13} \mathrm{C}$ NMR $(101$ $\left.\mathrm{MHz}, \mathrm{CDCl}_{3}\right) \delta: 158.3,153.1,148.7,146.9,144.8,142.8$, $130.9,130.4,129.5,128.8,128.1,128.0,127.6,115.7$, 114.4, 95.4, 63.4, 49.8, 20.8, 13.9; ESI-HRMS calcd for $\mathrm{C}_{20} \mathrm{H}_{17} \mathrm{~N}_{2} \mathrm{O}_{2}[\mathrm{M}+\mathrm{H}]^{+}$317.1285, found 317.1275.

\subsection{8 目标化合物 1 的合成}

11 经过两步文献 ${ }^{[8]}$ 报道的已知反应即可容易地最 终实现喜树碱 1 的全合成，表征数据与文献[8]一致.

辅助材料(Supporting Information) 化合物 9, 11, 16 20 的 ${ }^{1} \mathrm{HNMR}$ 谱和 ${ }^{13} \mathrm{CNMR}$ 谱图. 这些材料可以免费从 本刊网站(http://sioc-journal.cn/)上下载.

\section{References}

[1] Wall, M. E.; Wani, M. C.; Cook, C. E.; Palmer, K. H.; McPhail, A. T.; Sim, G. A. J. Am. Chem. Soc. 1966, 88, 3888.

[2] Moertel, C. G.; Schutt, A. J.; Reitemeier, R. J.; Hahn, R. G. Cancer Chemother. Rep. 1972, 56, 95.

[3] (a) Hsiang, Y. H.; Hertzberg, R.; Hecht, S.; Liu, L. F. J. Biol. Chem. 1985, 260, 14873.

(b) Hertzberg, R. P.; Busby, R. W.; Caranfa, M. J.; Holden, K. G.; John-son, R. K.; Hecht, S. M.; Kingsbury, W. D. J. Biol. Chem. 1990, 265, 19287.

(c) Pommier, Y.; Kohlhagen, G.; Kohn, K. W.; Leteurtre, F.; Wani, M. C.; Wall, M. E. Proc. Natl. Acad. Sci. U. S. A. 1995, 92, 8861.

[4] (a) Uehling, D. E.; Nanthakumar, S. S.; Croom, D.; Emerson, D. L.; Leitner, P. P.; Luzzio, M. J.; McIntyre, G.; Morton, B.; Profeta, S. J. Med. Chem. 1995, 38, 1106.

(b) Jew, S. S.; Kim, H. J.; Kim, M. G.; Roh, E. Y.; Cho, Y. S.; Kim, J. K.; Cha, K. H.; Lee, K. K.; Han, H. J.; Choi, J. Y.; Lee, H. Bioorg. Med. Chem. Lett. 1996, 6, 845.

(c) Pan, X. D.; Han, R.; Sun, P. Y. Bioorg. Med. Chem. Lett. 2003, 13, 3739 .

(d) Tangirala, R. S.; Dixon, R.; Yang, D. Z.; Ambrus, A.; Antony, S.; Agama, K.; Pommier, Y.; Curran, D. P. Bioorg. Med. Chem. Lett. 2005, 15, 4736 .

(e) Hutchinson, C. R. Tetrahedron 1981, 37, 1047.

(f) Du, W. Tetrahedron 2003, 59, 8649.

(g) Thomas, C. J.; Rahier, N. J.; Hecht, S. M. Bioorg. Med. Chem. 2004, 12, 1585 .

(h) Li, Q. Y.; Zu, Y. G.; Shi, R. Z.; Yao, L. P. Curr. Med. Chem. 2006, 13, 2021.

(i) Verma, R. P.; Hansch, C. Chem. Rev. 2009, 109, 213.

(j) Martino, E.; Volpe, S. D.; Terribile, E.; Benetti, E.; Sakaj, M.; Centamore, A.; Sala, A.; Collina, S. Bioorg. Med. Chem. Lett. 2017, 27, 701 .

(k) Pan, P.; Chen, J.; Li, M. Y.; Yu, H. D.; Zhao, J. J.; Ni, J.; Wang, X. W.; Sun, H. Y.; Tian, S.; Zhu, F.; Liu, F.; Huang, Y.; Hou, T. J. J. Med. Chem. 2018, 61, 8613.

[5] Kawato, Y.; Aonuma, M.; Hirota, Y.; Kuga, H.; Sato, K. Cancer Res. 1991, 51, 4187.

[6] Kingsbury, W. D.; Boehm, J. C.; Jakas, D. R.; Holden, K. G.;
Hecht, S. M.; Gallagher, G.; Caranfa, M. J.; Mccabe, F. L.; Faucette, L. F.; Johnson, R. K.; Hertzberg, R. P. J. Med. Chem. 1991, 34,98 .

[7] Ban, H. J.; Oh, I. J.; Kim, K. S.; Ju, J. Y.; Kwon, Y.S.; Kim, Y. I.; Lim, S. C.; Kim, Y. C. Tuberc. Respir. Dis. 2009, 66, 93

[8] Stork, G.; Schultz, A. G. J. Am. Chem. Soc. 1971, 93, 4074.

[9] For reviews, see: (a) Schultz, A. G. Chem. Rev. 1973, 73, 385. (b) Shamma, M.; Georgiev, V. St. J. Pharm. Sci. 1974, 63, 163. (c) Takayama, H.; Kitajima, M.; Aimi, N. J. Synth. Org. Chem. 1999, 57, 181

(d) Baurle, S.; Koert, U. In Organic Synthesis Highlights IV, Ed.: Schmalz, H. G., Wiley-VCH, Weinheim, 2000, p. 232

(e) Kawato, Y.; Terasawa, H. Prog. Med. Chem. 1997, 34, 69.

(f) Hutchinson, C. R. Tetrahedron 1981, 37, 1047.

(g) Thomas, C. J.; Rahier, N. J.; Hecht, S. M. Bioorg. Med. Chem. 2004, 12, 1585.

(h) Li, Q. Y.; Zu, Y. G.; Yao, L. P. Curr. Med. Chem. 2006, 13 2021.

(i) Liew, S. T.; Yang, L. X. Curr. Pharm. Des. 2008, 14, 1078

(j) Du, W. Tetrahedron 2003, 59, 8649.

(k) Chen, L.; Chen, F. E. Synlett 2017, 28, 1134

For recent research articles after 2017, see: (1) Wang, X. L.; Xu, L. J.; Xiong, F. J.; Wu, Y.; Chen, F. E. Tetrahedron: Asymmetry 2017 28,843

(m) Yuan, Y.; Dong, W. H.; Gao, X. S.; Xie, X. M.; Curran, D. P.; Zhang, Z. G. Chin. J. Chem. 2018, 36, 1035.

(n) Liu, Q.; Huang, G. X.; Liu, M. J.; Chen, F. E. Eur. J. Org. Chem. 2019, 6024

(o) Liu, Q.; Huang, G. X.; Liu, M. J.; Chen, F. E. Synthesis 2019 , 51,3506 .

(p) Liu, Q.; Liu, M. J.; Huang, G. X.; Chen, F. E. Tetrahedron 2019 , $75,2647$.

(q) Dong, W. H.; Yuan, Y.; Hu, B.; Gao, X. S.; Gao, H.; Xie, X. M.; Zhang, Z. G. Org. Lett. 2018, $20,80$.

[10] (a) Gaich, T.; Baran, P. S. J. Org. Chem. 2010, 75, 4657.

(b) Zheng, K.; Shen, D. F.; Zhang, B. B.; Hong, R. J. Org. Chem. 2020, 85,13818 .

(c) Vieira de Castro, T.; Yahiaoui, O.; Peralta, R. A.; Fallon, T.; Lee, V.; George, J. H. Org. Lett. 2020, 22, 8161.

[11] Wenkert, E.; Dave, K. G.; Lewis, R. G.; Sprague, P. W. J. Am. Chem. Soc. 1967, 89, 6741

[12] (a) Winterfeldt, E.; Rodunz, H. Chem. Commun. 1971, 374. (b) Winterfeldt, E.; Korth, T.; Pike, D.; Boch, M. Angew. Chem., Int. Ed. 1972, 11, 289.

[13] (a) Brown, R. T.; Leonard, J.; Sleigh, S. K. Phytochemistry 1978 , 17,899

(b) Brown, R, T.; Liu, J.; Santos, C. A. M. Tetrahedron Lett. 2000 , 41,859 .

[14] Nguyen, T. M.; Peixoto, S.; Ouairy, C.; Nguyen, T. D.; Bénéchie. M.; Marazano, C.; Michel, P. Synthesis 2010, 103.

[15] Yan, L. H.; Skiredj, A.; Dory, Y.; Delpech, B.; Poupon, E. Eur. J. Org. Chem. 2014, 4973.

[16] (a) Nuhant, P.; Raikar, S. B.; Wypych, J. C.; Delpech, B.; Mazarano, C. J. Org. Chem. 2009, 74, 9413.

(b) Overman, L. E.; Robichaud, A. J. J. Am. Chem. Soc. 1989, 111, 300 .

[17] Liu, G. S.; Dong, Q. L.; Yao, Y. S.; Yao, Z. J. Org. Lett. 2008, 10, 5393.

[18] Yuan, Y. H.; Han, X.; Zhu, F. P.; Tian, J. M.; Zhang, F. M.; Zhang, X. M.; Tu, Y. Q.; Wang, S. H.; Guo, X. Nat. Commun. 2019, 10 3394.

[19] Takayama, H.; Ishikawa, H.; Kurihara, M.; Kitajima, M.; Aimi, N.; Ponglux, D.; Koyama, F.; Matsumoto, K.; Moriyama, T.; Yamamoto, L. T.; Watanabe, K.; Murayama, T.; Horie, S. J. Med. Chem. 2002, 45, 1949.

[20] Thomas, O. P.; Zaparucha, A.; Husson, H. P. Eur. J. Org. Chem. 2002, 157.

[21] Li, K.; Ou, J. J.; Gao, S. H. Angew. Chem., Int. Ed. 2016, 55, 14778.

(Zhao, C.) 